\title{
Two-stage cracking of metallic bi-layers on polymer substrates under tension
}

\author{
B. Putz ${ }^{\mathrm{a}}$, C. May-Miller ${ }^{\mathrm{a}}$, V. Matl ${ }^{\mathrm{a}}$, B. Völker ${ }^{\mathrm{a}}$, D.M. Többens ${ }^{\mathrm{b}}$, C. Semprimoschnig ${ }^{\mathrm{c}}$, M.J. \\ Cordill $^{\mathrm{a}^{*}}$
}

${ }^{\text {a }}$ Erich Schmid Institute of Materials Science, Austrian Academy of Sciences, and Dept. Material Physics, Montanuniversität Leoben Jahnstraße 12, Leoben, Austria, 8700

${ }^{\mathrm{b}}$ Helmholtz-Zentrum Berlin für Materialien und Energie (HZB), Albert-Einstein-Str. 15, 12489 Berlin, Germany

${ }^{\mathrm{c}}$ European Space Research and Technology Centre (ESTEC), Keplerlaan 1, 2201 AZ Noordwijk, The Netherlands

*corresponding author: megan.cordill@oeaw.ac.at

\begin{abstract}
:
This study demonstrates two-stage cracking of an Inconel-silver film system on a flexible Teflon substrate under uniaxial tension. In situ fragmentation experiments revealed that primary fracture of the brittle 30nm Inconel overcoat induced brittle, secondary fracture of the normally ductile $150 \mathrm{~nm}$ silver base layer. Good correlation exists between the ratios of primary and secondary saturation crack spacing and individual layer thicknesses. Two-stage cracking was confirmed by cross-sectional analysis and explained by different steady state energy release rates of the individual layers. The results further illustrate how brittle layers are detrimental to the mechanical behavior of polymer-supported thin film multilayer structures.
\end{abstract}


Thin metal films on flexible polymer substrates facilitate a variety of innovative technologies, including flexible displays [1,2] or satellite insulation [3,4] by providing flexibility, weight-savings and a unique combination of material properties. The complex functionality of individual applications often requires a multilayer design [5-7] and the combination of different metal layers can lead to improved adhesion, corrosion resistance or thermal stability. Brittle interlayers [6,8] or overcoats are commonly used to improve adhesion or corrosion resistance of ductile metal layers. However, the failure behavior of ductile and brittle thin films on polymers is fundamentally different under tension. Brittle films fracture at low strains while ductile films typically thin locally (necking) and multilayers tend to take the failure behaviour of the most brittle layer when strained, bent or twisted $[9,10]$. In situ fragmentation testing is a common method to investigate the fracture behaviour of thin films on compliant substrates [11-14] where the uniaxial tensile test is combined with a surface imaging technique, such as optical microscopy (OM), confocal laser scanning microscopy (CLSM), or scanning electron microscopy (SEM) to observe failure of the film. More advanced techniques also include X-ray diffraction (XRD) and 4-point-probe resistance measurements to monitor the evolution of film lattice stress and electrical resistance, respectively, as a function of applied strain [15]. Characteristic values derived from this analysis (fracture strain, $\varepsilon_{\mathrm{f}}$, saturation cracks spacing, $\lambda_{\text {sat }}$ ) can be used to calculate interfacial properties or fracture toughness of films [16-19]. This study investigates the fragmentation behaviour of a brittle Inconel and ductile Ag bi-layer system on fluorinated ethylene propylene (FEP, Teflon $\left.{ }^{\circledR}\right)$ used as thermal insulation of second surface mirrors on satellites $[3,4]$. It will be shown that when the system comprises of a brittle overcoat film on a ductile layer, two-stage cracking is observed, which demands further investigation of brittle/ductile multilayer architectures.

Evaporated Ag films (nominal thickness 150nm) on a $50 \mu \mathrm{m}$ FEP substrate were supplied by Sheldahl Brand. To prevent the Ag from oxidizing a $275 \AA$ overcoat of Inconel was applied. The Ag layer has a nano-crystalline microstructure. The coherent domain size was measured with X-ray diffraction as $55 \pm 15 \mathrm{~nm}$, using the simple Scherrer-Equation and the advanced Williamson-Hall method [20,21]. Five diffraction peaks were used to estimate the grain size with both methods. For the Inconel overcoat the grain size is on the order of the film thickness. Rectangular samples were cut with a scalpel to the dimensions of $5 \times 40 \mathrm{~mm}$ for in situ fragmentation experiments, which were performed with an Anton Paar TS600 straining stage under an optical microscope (Olympus BX51) and a CLSM (Olympus LEXT 4100 OLS). In order to study the fracture of the Inconel and Ag layers independently, in situ OM straining experiments were performed looking onto the Inconel surface and focusing through the 
transparent FEP substrate onto the Ag layer. Samples were strained to maximum strains between $14-15 \%$ at a rate of $2 \mu \mathrm{m} / \mathrm{s}$ using pauses at pre-determined strains to allow for imaging of the film surface. Crack spacing analysis was performed with the OM and CLSM images with Image-J [22] and Gwyddion [23].

Synchrotron radiation (KMC-2 beamline [24], BESSY II, Berlin) was used to measure the fracture stress of the Ag film in situ using XRD and the $\sin ^{2} \psi$ method [25]. The longitudinal Ag lattice strain (parallel to the tensile direction) was measured in situ during continuous straining (Anton Paar TS600, $\varepsilon_{\max }=12 \%$, displacement rates of $1 \mu \mathrm{m} / \mathrm{s}$ and $2 \mu \mathrm{m} / \mathrm{s}$ ) in reflection geometry. The (111) reflections of the Ag layer were recorded simultaneously with a Bruker VÅNTEC 2000 detector using 5 different $\psi$ angles between 0 and 50 degrees, with an exposure time of $5 \mathrm{~s}$ and a beam wavelength of $0.177 \mathrm{~nm}$. A Pearson fit was applied to determine peak positions and peak widths. Film stresses were calculated using X-ray elastic constants (XECs) $\left(1 / 2 S_{2}\right)$ [26] for untextured (111) Ag reflections. XECs were calculated from single-crystal elastic constants assuming the Hill model with the software ElastiX [27]. The Inconel overcoat was too thin to receive a sufficient signal for $\sin ^{2} \psi$ analysis and the Inconel fracture stress was estimated from in situ CLSM experiments using Hooke's law $(\sigma=\varepsilon E$, with the elastic modulus of the Inconel $\left.E_{\text {inc }}=166 \mathrm{GPa}[28]\right)$.

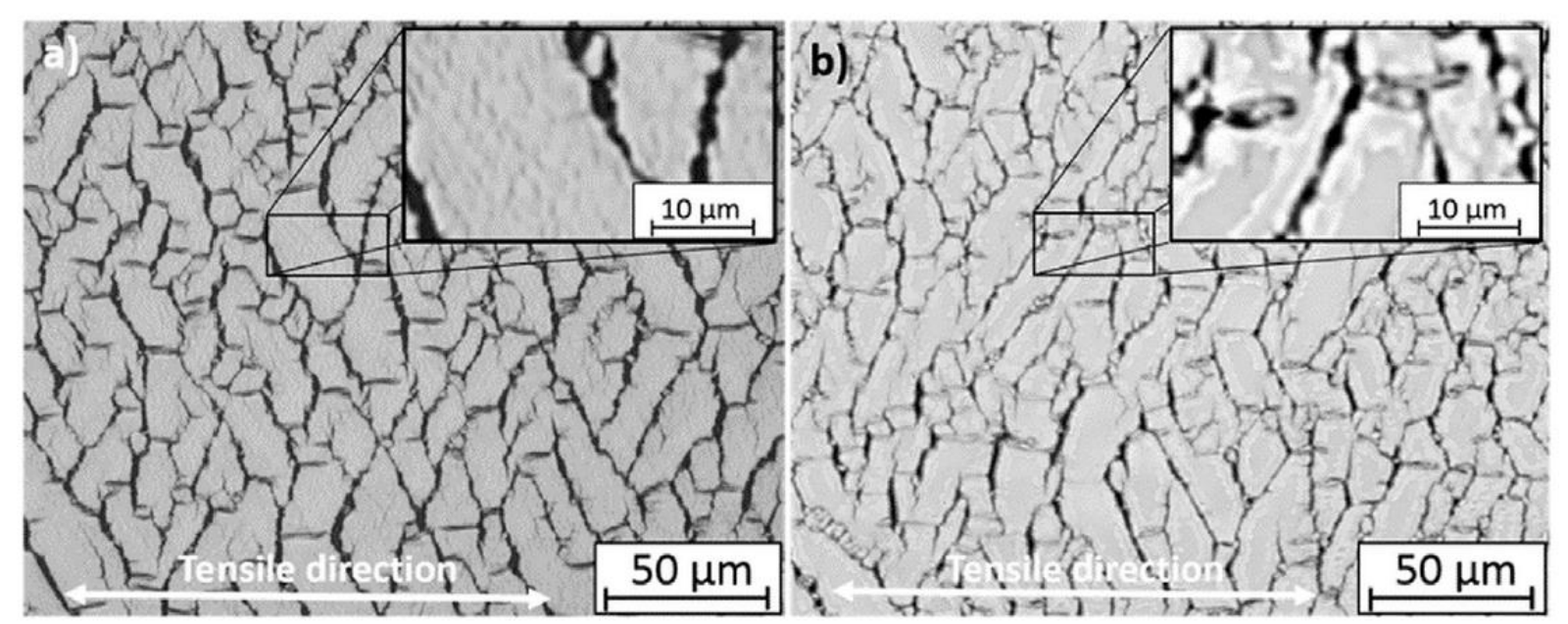

FIG. 1. In situ optical fragmentation analysis at $\varepsilon=15 \%$ revealing a two-stage cracking process. a) In the Inconel overcoat fine primary cracks (inset) and pronounced secondary cracks through both layers are visible. b) Only secondary cracks are visible in the Ag layer.

In situ optical fragmentation analysis from the Inconel side and Ag side (through the transparent FEP substrate) revealed a two-stage cracking process of the bi-layer perpendicular to the straining direction (Fig. 1). On the Inconel surface a fine pattern of primary cracks (inset of Fig. 1a) is present between the secondary cracks, which form through the Ag film. From the 
Ag side only secondary cracks are visible, indicating that the fine primary cracks are constrained to the Inconel overcoat. The average distance between secondary cracks measured at $15 \%$ strain is identical for the Inconel and Ag sides indicating that secondary cracks have formed through both layers. The small features parallel to straining direction visible in Fig. 1a and $1 \mathrm{~b}$ are tensile induced delaminations of the film at the Ag-FEP interface, which form around $4 \%$ strain. Buckling is compression-induced and a consequence of lateral contraction of the metal film on the polymer substrate during tensile straining. The tensile induced delamination phenomena has been described in $[29,30]$.
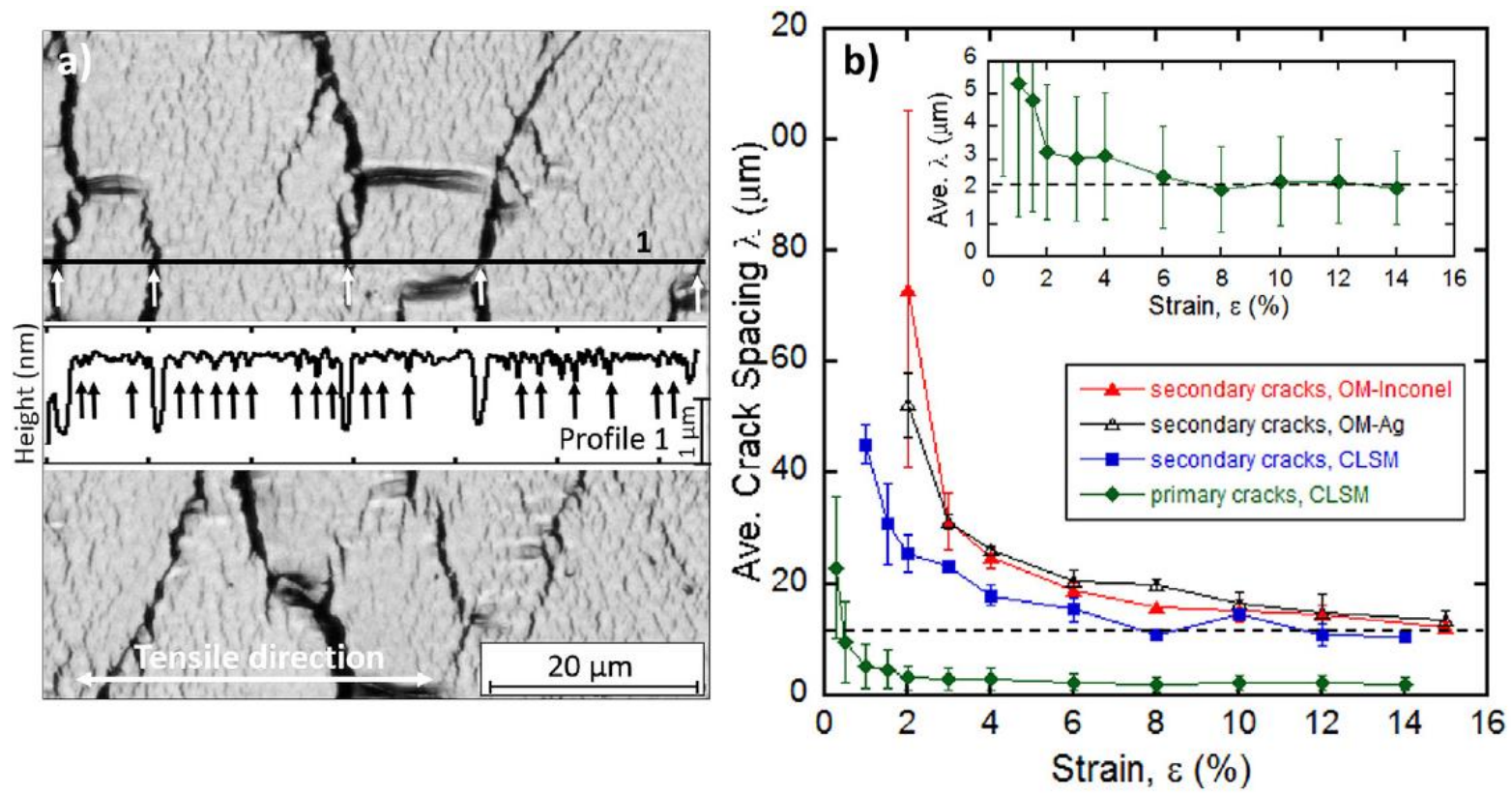

FIG. 2. a) CLSM laser intensity image $(\varepsilon=14 \%)$ and extracted height profile. Primary and secondary cracks are indicated with black and white arrows, respectively. b) Crack spacing as function of strain for primary (in situ CLSM, inset) and secondary cracks.

To fully investigate the evolution of primary and secondary cracking as a function of strain in situ CSLM experiments were performed. Fig. 2a shows a representative CLSM laser intensity image of the Inconel layer at $14 \%$ strain, with primary Inconel and secondary Ag cracks, indicated with black and white arrows, respectively. To measure the crack spacing of the primary and secondary cracks, surface profiles were extracted from the CLSM images. Primary cracks can be identified as small drops in the extracted profile (inset Fig. 2a) and the large drops correspond to secondary cracks. For each straining step, three profiles were extracted to calculate a mean value and standard deviation of the primary and secondary crack spacing. Fig. 2b summarizes the evolution of the primary and secondary crack spacing as a function of applied strain results from all in situ OM experiments. 
Primary cracks were first observed around $0.25 \%$ strain and the saturation regime was reached at about 4-6\% strain (inset Fig. 2b). The saturation spacing of the primary Inconel cracks was measured as $2.3 \pm 0.2 \mu \mathrm{m}$ (dotted line, inset Fig. 2b). Around $1 \%$ strain the second stage of cracking initiated, where individual primary cracks transformed into secondary cracks through the Ag layer. The fracture strain of the Ag film is much lower than what was observed for 100nm evaporated Ag and inkjet printed Ag, having fracture strains in the range of 5\% [31]. With increased strain the density of the secondary cracks increased as more primary cracks propagate through the Ag layer until a saturation state is reached around 8-10\% strain. The evolution of secondary cracks from the OM and CLSM experiments compare well to one another (Fig. 2b) and show the same trend of decreasing crack spacing with increasing strain. The secondary saturation crack spacing $(11.9 \pm 1.9 \mu \mathrm{m})$, indicated with a dotted line, is 5 times larger than the saturation spacing of the primary Inconel cracks $(2.3 \mu \mathrm{m})$ (ratio 5.2:1) and could be related to the film thickness ratio of 5:1.
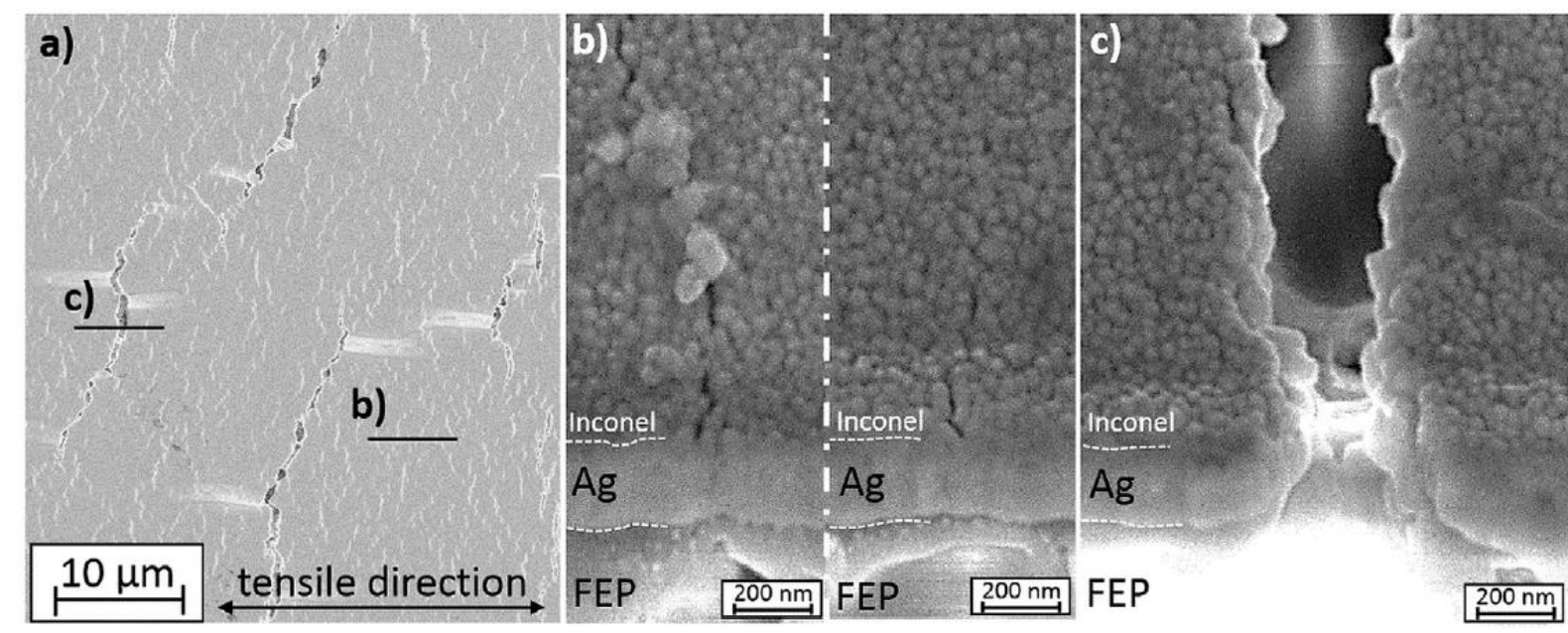

FIG. 3. Looking onto the strained Inconel surface $(\varepsilon=14 \%)$ (a) primary and secondary cracks are visible in the SEM micrograph. FIB cross-sections through both metal layers show that primary cracks (b) are constrained to the Inconel overcoat and secondary cracks (c) penetrate both metal layers.

To provide further evidence that primary cracks are only present in the Inconel overcoat, cross-sections were cut using focused ion beam (FIB). In Fig. 3a, the Inconel overcoat after straining to $14 \%$ is shown with visible primary and secondary cracks perpendicular to straining direction. Fig. $3 \mathrm{~b}$ and $3 \mathrm{c}$ show selected FIB cross-sections through both metal layers with primary and a secondary cracks, respectively. Primary cracks are constrained to the 30nm 
Inconel overcoat, while secondary cracks penetrate both metal layers, confirming the two-stage cracking mechanism.

The Ag stress evolution with the Inconel overcoat was determined from the lattice strains measured with in situ XRD and the $\sin ^{2} \psi$ analysis using the (111) Ag reflection (Fig. 4). Upon straining, the lattice strain increases and reaches a peak between $0.6 \%$ and $1.6 \%$ engineering strain due to secondary cracking of the Ag layer, which corresponds well to the transformation of primary into secondary cracks observed earlier and is considered the failure strain. After the peak strain is reached (ave. peak stress $310 \mathrm{MPa}$ ) the lattice strain decreases rapidly, reaching a plateau around $6 \%$ strain. The plateau corresponds to the beginning of the saturation regime of the secondary cracking process. During unloading, the lattice strain falls into the compressive regime. The evolution of the measured Ag lattice strain with the Inconel overcoat is similar to that found for gold [15] and copper films [6], both with a thin Cr interlayer and provides direct evidence that a brittle layer at any position induces brittle behaviour in normally ductile metals. It is also believed that the two-stage cracking also occurs when the brittle interlayer is next to substrate, even though it cannot be directly observed.

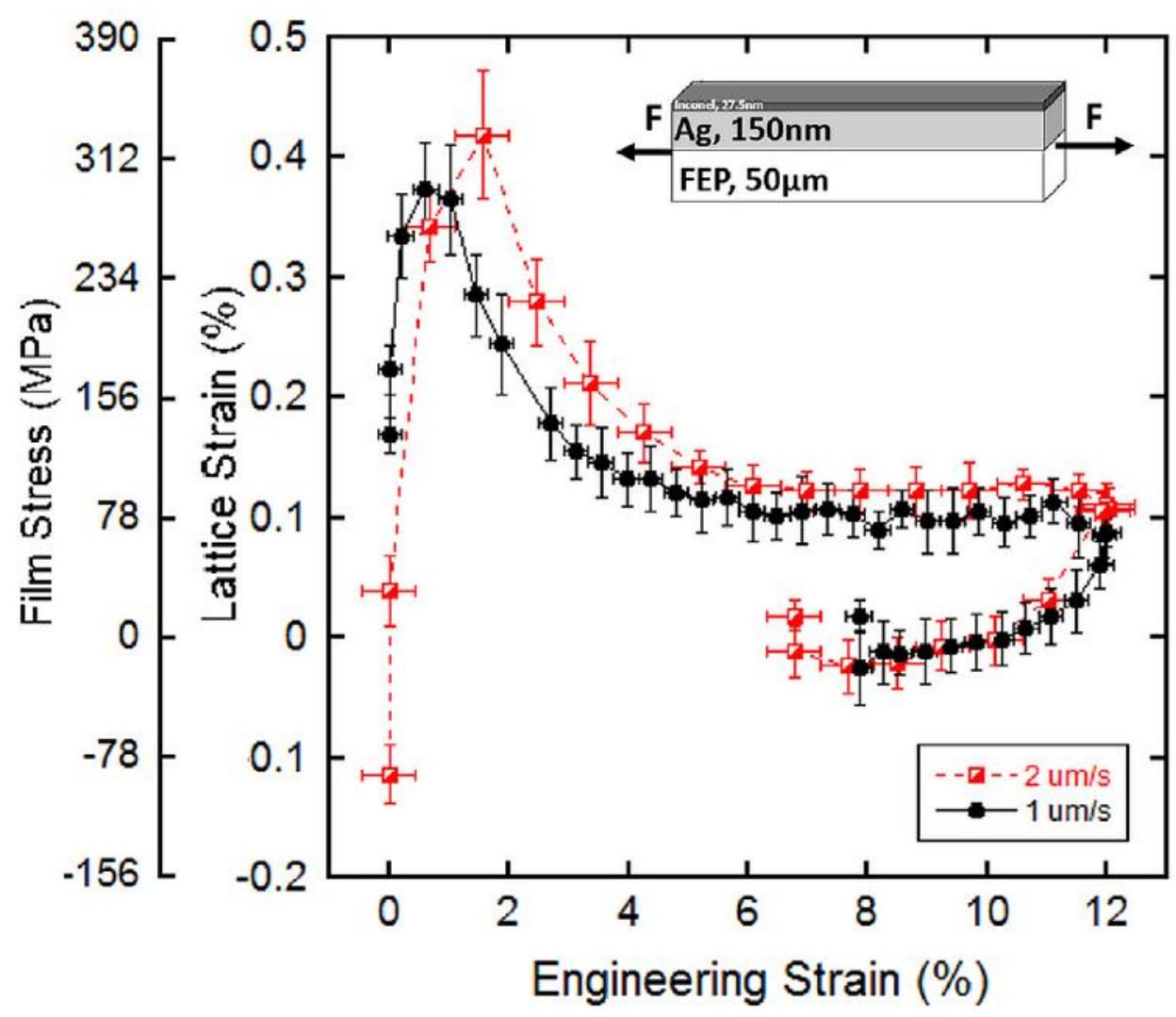

FIG. 4. In situ XRD of the Ag lattice strain using the (111) Ag reflection. Fracture of the Ag layer starts after the lattice strain reaches a peak between $0.6 \%$ and $1.6 \%$ engineering strain. 
Because the fracture of both layers can be observed or directly measured, the steady state energy release rate, $G_{\mathrm{ss}}$, of the Inconel and the Ag layer can be evaluated with the approach described in Refs. [18,19,32]. Using the fracture stress, $\sigma$, film thickness, $h$, and elastic properties of the films, $E_{f}$ and $v_{f}, G_{\mathrm{ss}}$ is determined with Eqn. 1

$$
G_{S S}=\frac{\pi \sigma^{2} h\left(1-v_{f}^{2}\right)}{2 E_{f}} g(\alpha, \beta),
$$

where $g(\alpha, \beta)$ is a dimensionless parameter based on the Dundur's parameters of $\alpha$ and $\beta$ $[19,33]$. Due to the fact that it was observed that the Inconel and Ag layers fracture at different strains, the $G_{\mathrm{ss}}$ can be evaluated for the primary Inconel cracking $\left(\sigma_{\mathrm{Inc}}=415 \mathrm{MPa}\right)$ and the secondary Ag cracking $\left(\sigma_{\mathrm{Ag}}=310 \mathrm{MPa}\right)$. Using the elastic constants [34] for the Inconel $\left(v_{\text {Inc }}=0.31\right), \mathrm{Ag}\left(E_{\mathrm{Ag}}=74 \mathrm{GPa} ; v_{\mathrm{Ag}}=0.37\right)$, and FEP $\left(E_{\mathrm{FEP}}=550 \mathrm{MPa} ; v_{\mathrm{FEP}}=0.46\right)$ substrate, the Dundur's parameter $\alpha$ was determined for the Inconel-Ag interface $(\alpha=0.3635)$ and the Ag-FEP interface ( $\alpha=0.9839)$. The dimensionless parameters $g(\alpha, \beta)$ were obtained from Table 2 found in Beuth [19], which also shows that $\beta$ has a negligible influence on $g(\alpha, \beta)$. Primary Inconel $G_{\mathrm{ss}}$ was calculated to be $0.065 \mathrm{Jm}^{-2}$ using 22.68 as the dimensionless parameter. The secondary $\mathrm{Ag} G_{\mathrm{ss}}$ was found to be $6 \mathrm{Jm}^{-2}$ using the dimensionless parameter of 1.6. The primary Inconel energy release rate is 100 times lower than the secondary Ag energy release rate, illustrating that the Inconel layer fractures first (primary cracking) because it has a lower crack extension force compared to the Ag. The use of this model quantitatively describes the observed behaviour and leads to the conclusion that the primary cracks act as stress concentrators forcing cracks through the Ag film.

It has been demonstrated that transparent polymer substrates are ideal to investigate the fracture behavior of multilayer film systems using in situ fragmentation experiments with a fast technique such as OM, while avoiding detrimental electron interactions with the polymer during the straining. Primary cracks first formed in the brittle Inconel overcoat and acted as stress concentrators causing the normally ductile $\mathrm{Ag}$ film to fracture (secondary cracks). Cracking of both layers at low strains ( $\varepsilon_{\text {Prim }}=0.25 \% ; \varepsilon \mathrm{Sec}=1 \%$ ) deteriorates the functionality of the material and has fatal consequences for the designated application as a second surface mirror or any flexible application. Both fracture strains can be considered a figure of merit. The cracks in the Ag layer directly influence the reflectivity of the metallization, a critical material property for sufficient insulation and cracking of the Inconel overcoat deteriorates the corrosion resistance and long term performance of the surface mirror. Investigation of reflectivity and corrosion resistance as a function of crack density is proposed as a subject of continuing research. The two-stage cracking has also been observed in simulations for ductile $\mathrm{Cu}$ films with a $\mathrm{Cr}$ 
interlayer [6]. What the results demonstrate is that when bi-layer systems are comprised of at least one brittle layer, either as an adhesion layer or protective overcoat, the film system will behave in a brittle manner unless the ductile layer is of a sufficient thickness to allow for significant deformation. This is critical for all flexible applications requiring a ductile-brittle multilayer design. To delay fracture of the Ag layer, an increased layer thickness is proposed with a film thickness ratio greater than 5:1. Determination of the minimum required thickness of the ductile layer to suppress the influence of the overcoat is an avenue of future investigation. It is believed that a ductile-brittle film thickness ratio of 10:1 or 20:1 [6] is ideal. Internal stress tuning or a change of material to a more ductile corrosion protection layer or replacement of $\mathrm{Ag}$ with inert $\mathrm{Au}$, are also conceivable. However, these fundamental changes would certainly require thorough investigations to ensure that the initially desired thermo-optical properties are still obtained.

\section{Acknowledgements}

Partial funding of the research study has been provided by the European Space Agency (project 4000111838/14/NL/PA) and by the Helmholtz-Zentrum Berlin (HZB) (project no. 15101992ST and 1610331-ST/R).

\section{References}

[1] A. Nathan, B.R. Chalamala, Proc. IEEE 93 (2005) 1235-1238.

[2] A. Nathan, B.R. Chalamala, Proc. IEEE 93 (2005) 1391-1393.

[3] J.B. Heaney, Goddard Sp. Flight Cent. Rep. (1974).

[4] J.A. Dever, K.K. De Groh, B.A. Banks, J.A. Townsend, High Perform. Polym. Polym 11 (1999) 123-140.

[5] P.A. Gruber, E. Arzt, R. Spolenak, J. Mater. Res. 24 (2009) 1906-1918.

[6] V.M. Marx, F. Toth, A. Wiesinger, J. Berger, C. Kirchlechner, M.J. Cordill, F.D. Fischer, F.G. Rammerstorfer, G. Dehm, Acta Mater. 89 (2015) 278-289.

[7] X.F. Zhu, B. Zhang, J. Gao, G.P. Zhang, Scr. Mater. 60 (2009) 178-181.

[8] B. Putz, R.L. Schoeppner, O. Glushko, D.F. Bahr, M.J. Cordill, Scr. Mater. 102 (2015) 23-26.

[9] L.R. Brandt, E. Salvati, C. Papadaki, H. Zhang, S. Ying, E. Le Bourhis, I. Dolbnya, T. Sui, A.M. Korsunsky, Surf. Coatings Technol. 320 (2016) 158-167.

[10] M.N. Polyakov, J. Lohmiller, P.A. Gruber, A.M. Hodge, Adv. Eng. Mater. 15 (2014) 810-814.

[11] M.J. Cordill, JOM 62 (2010) 9-14. 
[12] T. Li, Z.Y. Huang, Z.C. Xi, S.P. Lacour, S. Wagner, Z. Suo, Mech. Mater. 37 (2005) 261-273.

[13] T. Lacroix, B. Tilmans, R. Keunings, M. Desaeger, I. Verpoest, Compos. Sci. Technol. 43 (1992) 379-387.

[14] T. Lacroix, R. Keunings, M. Desaeger, I. Verpoest, J. Mater. Sci. 30 (1995) 683-692.

[15] B. Putz, O. Glushko, V.M. Marx, C. Kirchlechner, D. Toebbens, M.J. Cordill, MRS Adv. 1 (2016) 773-778.

[16] H.L. Cox, Br. J. Appl. Phys. 3 (1952) 72-79.

[17] D.C. Agrawal, R. Raj, Acta Metall. 37 (1989) 1265-1270.

[18] T. Ye, Z. Suo, A.G. Evans, Int. J. Solids Struct. 29 (1992) 2639-2648.

[19] J.L. Beuth, Int. J. Solids Struct. 29 (1992) 1657-1675.

[20] G.. Williamson, W.. Hall, Acta Metall. 1 (1953) 22-31.

[21] W.H. Hall, Proc. Phys. Soc. London 62 (1949) 741-743.

[22] M.D. Abràmoff, P.J. Magalhães, S.J. Ram, Biophotonics Int. 11 (2004) 36-41.

[23] D. Nečas, P. Klapetek, Open Phys. 10 (2012) 181-188.

[24] D. Többens, S. Zander, J. Large-Scale Res. Facil. 2 (2016) 1-6.

[25] L. Spieß, G. Teichert, R. Schwarzer, H. Behnken, C. Genzel, Teubner, Wiesbad. (2005).

[26] I.C. Noyan, J.B. Cohen, Residual Stress: Measurement by Diffraction and Interpretation, Springer-Verlag, 2013.

[27] H. Wern, N. Koch, T. Maas, in:, Mater. Sci. Forum, 2002, pp. 127-132.

[28] G. Fleury, C. Malhaire, C. Populaire, M. Verdier, A. Devos, P.L. Charvet, J.P. Polizzi, Sensors Actuators, B Chem. 126 (2007) 48-51.

[29] M.J. Cordill, F.D. Fischer, F.G. Rammerstorfer, G. Dehm, Acta Mater. 58 (2010) 5520-5531.

[30] F. Toth, F.G. Rammerstorfer, M.J. Cordill, F.D. Fischer, Acta Mater. 61 (2013) 24252433.

[31] O. Glushko, A. Klug, E.J.W. List-Kratochvil, M.J. Cordill, J. Mater. Res. 32 (2017) 1760-1769.

[32] J.W. Hutchinson, Z. Suo, Adv. Appl. Mech. 29 (1992) 63-191.

[33] J. Dundurs, J. Appl. Mech. 36 (1969) 650-652.

[34] W.D. Callister, Materials Science and Engineering: An Introduction, 5th Editio, John Wiley \& Sons, Inc., New York, 2000. 